Case Report

\title{
A Rare Case of ARDS Caused by Bupropion Inhalation and Treated with Noninvasive Ventilation
}

\author{
Yousif Al-Saiegh $\mathbb{D},{ }^{1}$ Jenna Spears $\mathbb{D}^{1},{ }^{1}$ Pieter S. De Klerk, ${ }^{1}$ Joshua Hitchings, ${ }^{1}$ \\ Christopher Lee $\mathbb{1}^{1},{ }^{1}$ and Tamara $\mathrm{Mahr}^{2}$ \\ ${ }^{1}$ Department of Medicine, Pennsylvania Hospital, University of Pennsylvania Health System (UPHS), Philadelphia PA, USA \\ ${ }^{2}$ Department of Pulmonary/Critical Care, Pennsylvania Hospital, University of Pennsylvania Health System (UPHS), \\ Philadelphia PA, USA
}

Correspondence should be addressed to Yousif Al-Saiegh; yousif.al-saiegh@pennmedicine.upenn.edu

Received 29 January 2020; Accepted 23 March 2020; Published 28 May 2020

Academic Editor: Mabrouk Bahloul

Copyright (c) 2020 Yousif Al-Saiegh et al. This is an open access article distributed under the Creative Commons Attribution License, which permits unrestricted use, distribution, and reproduction in any medium, provided the original work is properly cited.

Acute respiratory distress syndrome, characterized by the Berlin criteria, is associated with a high mortality rate. Its treatment includes addressing the underlying etiology, general supportive measures, and achievement of effective oxygenation. New key data indicates that in a subset of patients, noninvasive ventilation techniques can be a therapeutic and equivalent alternative to traditional invasive ventilation. We present a rare case of ARDS triggered by nasal bupropion inhalation and effectively treated with noninvasive positive pressure ventilation resulting in complete resolution.

\section{Introduction}

Acute respiratory distress syndrome (ARDS) incorporates a cluster of clinical features including shortness of breath and tachypnea and is defined by the Berlin criteria as having an acute onset with the development of hypoxemia and bilateral pulmonary opacities on radiographic imaging [1]. There are many causes of ARDS; however, most often, it is triggered by infections, blood transfusions, direct lung injury, and toxins [2]. Treatment includes removal of the inciting cause, supportive therapy, and the attainment of sufficient blood oxygenation. Adequate oxygenation in ARDS is often achieved by endotracheal intubation and mechanical ventilation. Noninvasive positive pressure ventilation (NIPPV) has been less frequently indicated as an alternative form of adequate oxygenation. Typically, its use is focused and intended on preventing complications that are associated with invasive ventilation such as barotrauma, vocal cord injury, and ventilator-associated pneumonia [3].

Bupropion is an atypical oral antidepressant medication commonly used to treat depression, tobacco dependence, obesity, and hypoactive sexual disorder. Its mechanism of action involves the inhibition and reuptake of norepinephrine and dopamine which can induce a state of euphoria. Because of these effects, bupropion has been reported to be abused recreationally $[4,5]$. We describe a case of ARDS induced by bupropion inhalation that was treated with NIPPV.

\section{Case Presentation}

A 30-year-old male with a past medical history significant for polysubstance abuse presented to the emergency department (ED) two hours after ingesting $90 \mathrm{mg}$ of oxycodone and $30 \mathrm{mg}$ of diazepam along with intranasal bupropion. On arrival, he complained of extreme fatigue, respiratory distress, and confusion.

In the ED, he was noted to be lethargic and short of breath. Initial vital signs revealed heart rate of 104 beats per minute, respiratory rate of 35 per minute, and $\mathrm{O}_{2}$ saturation of $75 \%$ on room air. Respiratory exam revealed diffuse bilateral coarse breath sounds on inspiration and expiration. His laboratory results were notable for a leukocytosis of $14.1 \mathrm{~K}$, creatinine of $1.25 \mathrm{~g} / \mathrm{dl}$, lactate of 4.1 , and an elevated procalcitonin level. Venous blood gas analysis showed a $\mathrm{pH}$ of 


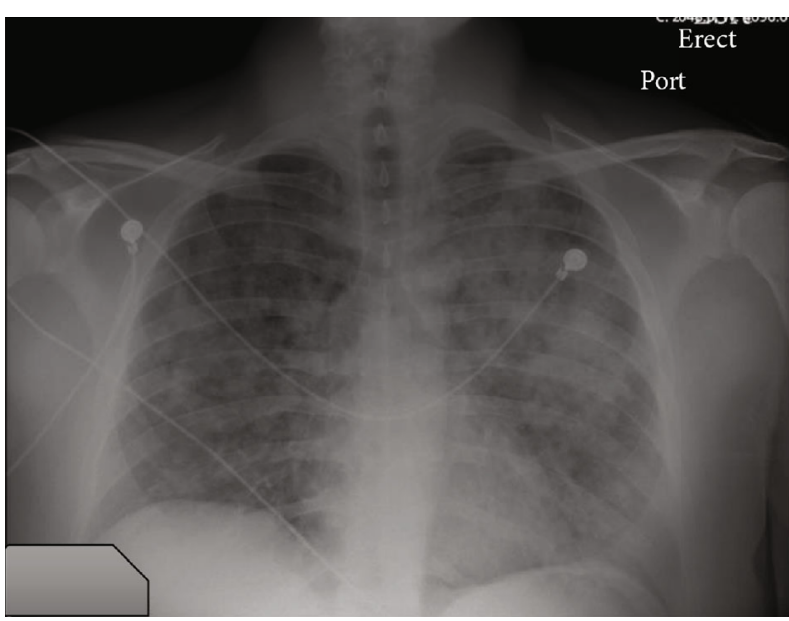

(a)

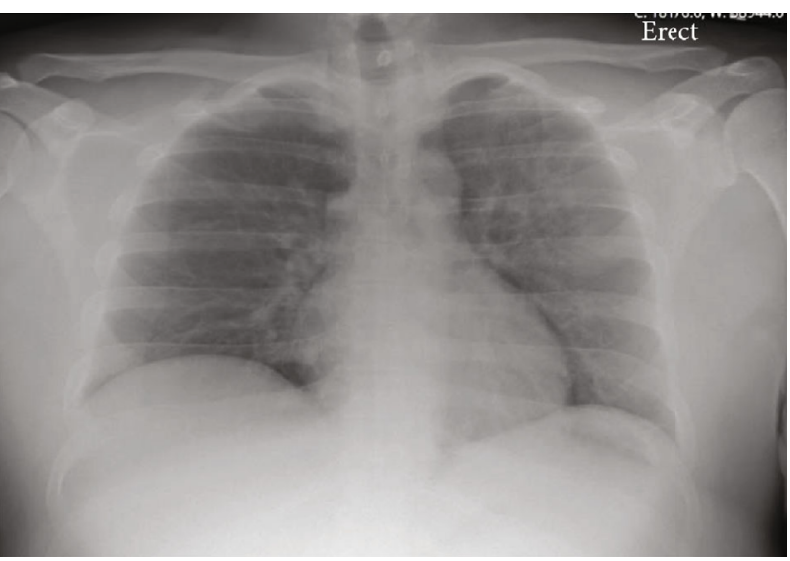

(b)

Figure 1: (a) Single-view anterior-posterior chest X-ray on day \#1 showed diffuse bilateral lung opacities. (b) Repeat single-view anterior-posterior chest X-ray on day \#6 showed decreased airspace opacities.

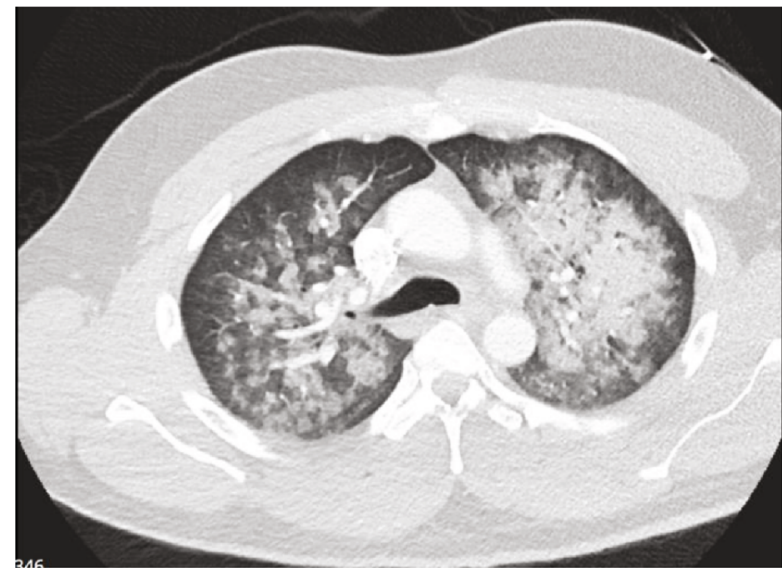

Figure 2: Contrast-enhanced computed tomography of the chest on day \#2 shows diffuse bilateral airspace disease characterized by groundglass and consolidative opacities, with relative peripheral sparing and perihilar predominance. No pleural effusions or pneumothorax.

7.18 with a $\mathrm{pCO}_{2}$ of $51 \mathrm{mmHg}$. Chest X-ray (CXR) demonstrated diffuse bilateral alveolar infiltrates (Figure 1(a)). Computed tomography (CT) of the chest showed diffuse bilateral airspace disease characterized by groundglass and consolidative opacities with relative peripheral lung sparing and perihilar predominance (Figure 2 ). He received naloxone with mild improvement in mental status and was initiated on Bilevel Positive Airway Pressure (BiPAP) with an inspiratory positive airway pressure (IPAP) of $15 \mathrm{~cm} \mathrm{H}_{2} \mathrm{O}$, expiratory positive airway pressure (EPAP) of $5 \mathrm{~cm} \mathrm{H}_{2} \mathrm{O}$ with $40 \%$ fraction of inspired oxygen $\left(\mathrm{FiO}_{2}\right)$. Empiric broad spectrum antibiotics including vancomycin, cefepime, and metronidazole were initiated along with intravenous methylprednisolone $40 \mathrm{mg}$ every 12 hours.

Initially while on BIPAP, he remained tachypneic and tachycardic. His respiratory rate improved over the following 6 hours. He was gradually weaned off BiPAP to nasal cannula oxygen over the course of 36 hours while receiving ongoing corticosteroid and antibiotic therapy.

Repeat CXR on hospital day \#6 showed markedly improved bilateral airspace opacities (Figure 1(b)). After 6 days, he was discharged in stable condition without requiring supplemental oxygen.

\section{Discussion}

ARDS is associated with a high mortality that has declined from over $50 \%$ to $30 \%$ over the last four decades $[6,7]$. This is primarily due to implementation of lung-protective ventilation protocols and intensified research after formation of the National Heart, Lung, and Blood Institute (NHLBI) ARDS network $[8,9]$. This case highlights a mild manifestation of ARDS as a result of bupropion inhalation, an exceedingly rare etiology.

The Food and Drug Administration (FDA) classifies bupropion as a psychiatric medication with low abuse potential [10]. However, several case reports and studies have indicated increasing recreational use of bupropion mostly intravenously or intranasally [11-13]. Lewis et al. conducted a review on bupropion inhalation in a total of 67 patients. Seizures were noted as a common adverse effect occurring in $30 \%$ of patients. Acute lung toxicity was not reported as a complication [14]. We were not able to find a second reported case of bupropion-inhalation-induced ARDS.

Endotracheal intubation and mechanical ventilation are mostly required to ensure adequate oxygenation, but this was avoided in this patient as we maintained adequate oxygenation with BiPAP alone. NIPPV is advantageous in such patients as it does not expose them to the potential complications of invasive ventilation and may shorten their hospital length of stay [3]. There is, however, an ongoing debate concerning the most effective mode of NIPPV $[15,16]$. Recent studies show that noninvasive ventilation can be used in mild cases of ARDS with acute nonhypercapnic hypoxemic respiratory failure [17]. In these cases, BiPAP via facemask is the most commonly used strategy [18]. Another approach with 
high-flow nasal cannula was shown to have a similar degree of treatment failure and incidence of subsequent intubation as BiPAP. High-flow nasal cannula, however, was associated with decreased 90-day mortality as compared to BiPAP [19]. A randomized, single-center trial by Pohlman et al. showed that delivering noninvasive ventilation via helmet instead of by facemask was associated with a significant reduction in intubations. There was also a decrease in intensive care unit length of stay and mortality at 90 days [20]. However, a subset analysis of the observational "LUNG-SAFE" study for patients with severe ARDS (defined as a $\mathrm{PaO}_{2} / \mathrm{FiO}_{2}$ ratio below $150 \mathrm{mmHg}$ ) showed increased mortality with noninvasive ventilation [21].

Per our review, data on the use of noninvasive ventilation in the management of ARDS remains inconclusive and conflicting. NIPPV may decrease the incidence of ventilatorassociated complications; however, given that its efficacy is not well established, patients with ARDS should still be treated in a critical care setting for close monitoring with invasive ventilation available on standby.

\section{Conclusion}

Advances in the treatment of the underlying etiologies and improvement in mechanical ventilation strategies have led to a decrease in the overall mortality associated with ARDS. Despite these developments, it remains a diagnosis with an exceedingly high mortality. New emerging data indicates that NIPPV can be a beneficial and equivalent approach for a subset of patients with ARDS, although the optimal type of noninvasive ventilation and patient group that would benefit most is yet to be determined.

\section{Conflicts of Interest}

All authors declare no conflict of interest.

\section{References}

[1] The ARDS Definition Task Force, "Acute respiratory distress syndrome: the Berlin definition," JAMA, vol. 307, pp. 25262533, 2012.

[2] E. Fan, D. Brodie, and A. S. Slutsky, "Acute respiratory distress syndrome: advances in diagnosis and treatment," JAMA, vol. 319, no. 7, pp. 698-710, 2018.

[3] A. Taha, E. Larumbe-Zabala, A. Abugroun, A. Mohammedzein, M. T. Naguib, and M. Patel, "Outcomes of noninvasive positive pressure ventilation in acute respiratory distress syndrome and their predictors: a national cohort," Critical Care Research and Practice, vol. 2019, Article ID 8106145, 8 pages, 2019.

[4] H. R. Arias, "Is the inhibition of nicotinic acetylcholine receptors by bupropion involved in its clinical actions?," The International Journal of Biochemistry \& Cell Biology, vol. 41, no. 11, pp. 2098-2108, 2009.

[5] B. Langguth, G. Hajak, M. Landgrebe, and W. Unglaub, "Abuse potential of bupropion nasal insufflation: a case report," Journal of Clinical Psychopharmacology, vol. 29, no. 6, pp. 618-619, 2009.

[6] J. A. Milberg, D. R. Davis, K. P. Steinberg, and L. D. Hudson, "Improved survival of patients with acute respiratory distress syndrome (ARDS): 1983-1993," JAMA, vol. 273, no. 4, pp. 306-309, 1995.

[7] D. G. Ashbaugh, D. B. Bigelow, T. L. Petty, and B. E. Levine, "Acute respiratory distress in adults," The Lancet, vol. 2, no. 7511, pp. 319-323, 1967.

[8] M. B. P. Amato, C. S. V. Barbas, D. M. Medeiros et al., "Effect of a protective-ventilation strategy on mortality in the acute respiratory distress syndrome," The New England Journal of Medicine, vol. 338, no. 6, pp. 347-354, 1998.

[9] B. T. Thompson and G. R. Bernard, "ARDS network (NHLBI) studies: successes and challenges in ARDS clinical research," Critical Care Clinics, vol. 27, no. 3, pp. 459-468, 2011.

[10] Substance abuse treatment for persons with HIVIAIDS, Substance Abuse and Mental Health Services Administration (US), Rockville, 2000.

[11] N. Stall, J. Godwin, and D. Juurlink, "Bupropion abuse and overdose," CMAJ, vol. 186, no. 13, p. 1015, 2014.

[12] G. L. Stassinos and W. Klein-Schwartz, "Bupropion "abuse" reported to US poison centers," Journal of Addiction Medicine, vol. 10, no. 5, pp. 357-362, 2016.

[13] P. Starr, W. Klein-Schwartz, H. Spiller, P. Kern, S. E. Ekleberry, and S. Kunkel, "Incidence and onset of delayed seizures after overdoses of extended-release bupropion," The American Journal of Emergency Medicine, vol. 27, no. 8, pp. 911-915, 2009.

[14] J. C. Lewis, M. E. Sutter, T. E. Albertson, K. P. Owen, and J. B. Ford, "An 11-year review of bupropion insufflation exposures in adults reported to the California Poison Control System," Clinical Toxicology, vol. 52, no. 9, pp. 969-972, 2014.

[15] J. Messika, K. Ben Ahmed, S. Gaudry et al., "Use of high-flow nasal cannula oxygen therapy in subjects with ARDS: a 1-year observational study," Respiratory Care, vol. 60, no. 2, pp. 162-169, 2015.

[16] X. Ou, Y. Hua, J. Liu, C. Gong, and W. Zhao, "Effect of highflow nasal cannula oxygen therapy in adults with acute hypoxemic respiratory failure: a meta-analysis of randomized controlled trials," CMAJ, vol. 189, no. 7, pp. E260-E267, 2017.

[17] Q. Zhan, B. Sun, L. Liang et al., "Early use of noninvasive positive pressure ventilation for acute lung injury: a multicenter randomized controlled trial," Critical Care Medicine, vol. 40, no. 2, pp. 455-460, 2012.

[18] M. Kredel, D. Bierbaum, C. Lotz, J. Kustermann, N. Roewer, and R. M. Muellenbach, "Therapy of acute respiratory distress syndrome : survey of German ARDS centers and scientific evidence," Anaesthesist, vol. 64, no. 4, pp. 277-285, 2015.

[19] J. P. Frat, A. W. Thille, A. Mercat et al., "High-flow oxygen through nasal cannula in acute hypoxemic respiratory failure," The New England Journal of Medicine, vol. 372, no. 23, pp. 2185-2196, 2015.

[20] B. K. Patel, K. S. Wolfe, A. S. Pohlman, J. B. Hall, and J. P. Kress, "Effect of noninvasive ventilation delivered by helmet vs face mask on the rate of endotracheal intubation in patients with acute respiratory distress syndrome: a randomized clinical trial," JAMA, vol. 315, no. 22, pp. 2435-2441, 2016.

[21] G. Bellani, J. G. Laffey, T. Pham et al., "Noninvasive ventilation of patients with acute respiratory distress syndrome. Insights from the LUNG SAFE study," American Journal of Respiratory and Critical Care Medicine, vol. 195, no. 1, pp. 67-77, 2017. 